\title{
OPPENHEIMER AND BONDI LIMITS: THE CASE OF THE SINGULAR CONFORMAL CURVATURE*
}

\author{
Andrzej Woszczyna, Sebastian Kubis
}

Institute of Physics, Tadeusz Kościuszko Cracow University of Technology

Podchorążych 1, 30-084 Kraków, Poland

\section{WojcIECh Czaja}

Copernicus Center for Interdisciplinary Studies

Sławkowska 17, 31-016 Kraków, Poland

Zdzisław A. Golda, Piotr Plaszczyk

Astronomical Observatory, Jagiellonian University

Orla 171, 30-244 Kraków, Poland

\section{MAREK KutscherA}

The Marian Smoluchowski Institute of Physics, Jagiellonian University Łojasiewicza 11, 30-348 Kraków, Poland

(Received October 22, 2015)

Spherically symmetric, gravitating configurations built of the regularly distributed gas in equilibrium still admit a singular Weyl curvature. In contrast to the Black Holes, the singularity located in the center of the gas cloud is naked - forms the repulsive point-like gravity source. Configurations of this kind can exceed both the Oppenheimer limit and the Bondi limit.

DOI:10.5506/APhysPolB.46.2357

PACS numbers: 04.20.Cv, 04.20.Dw, 04.25.dc

* Presented by A. Woszczyna at the XXXIX International Conference of Theoretical Physics "Matter to the Deepest", Ustron, Poland, September 13-18, 2015. 


\section{Introduction: the role of conformal curvature}

The Ricci curvature is coupled to matter via the Einstein equations. It is uniquely defined when the distribution of matter is specified. On the contrary, the Weyl tensor is a "free parameter" of the theory and can be chosen arbitrary - independently of the matter distribution. The freedom to arbitrary setting the conformal curvature is seldom disclosed in astrophysical investigations, where the symmetry of the energy-momentum tensor is usually replicated to metric tensor. This procedure does not result from the general relativity, neither from observations. Instead, it constitutes an additional constraint, and eventually results in the loss of generality.

Although the source of the Weyl tensor is not specified, this quantity has an important geometrical and physical meaning [1]. The Weyl tensor itself is the source of the geodesic deviation. Conformal curvature acts in the way similar to the tidal forces [1, 2], as well as it contributes to the ADM mass of spherical bodies [3].

\section{Nakedly-singular gaseous spheres in equilibrium}

In this paper, we discuss static, spherically symmetric configurations with the regularly distributed gas described by the hydrodynamic energymomentum tensor. Following the classical Oppenheimer-Volkoff approach [4], we assume the same symmetry for both the energy-momentum tensor and the metric tensor

$$
d s^{2}=-A(r) c^{2} d t^{2}+\left(1-2 \frac{G}{c^{2}} \frac{M(r)+M_{g}}{r}\right)^{-1} d r^{2}+r^{2}\left(d \vartheta^{2}+\sin ^{2} \vartheta d \varphi^{2}\right) .
$$

However, we relax the assumption of the metric tensor regularity $[4,5]$. The problem differs from the classical TOV problem in non-vanishing constant of integration $M_{g}$ which is explicitly present in the metrics (1). The Einstein equations $G_{\mu \nu}=\kappa T_{\mu \nu}$ reduce to non-autonomous dynamical system

$$
\begin{aligned}
M^{\prime}(r) & =4 \pi r^{2} \rho(r), \\
p^{\prime}(r) & =-G\left(c^{2} \rho(r)+p(r)\right) \frac{M(r)+M_{g}+4 \pi r^{3} p(r) c^{-2}}{r\left(r-2 G\left(M(r)+M_{g}\right) c^{-2}\right)} .
\end{aligned}
$$

By computing the Carminati-McLenaghan invariants $[6,7]$

$$
\begin{aligned}
\mathcal{R}_{1} & =3^{1 / 3} \mathcal{R}_{2}{ }^{2 / 3}, \\
\mathcal{R}_{3} & =\frac{7 \mathcal{R}_{2}{ }^{4 / 3}}{43^{1 / 3}}, \\
\mathcal{W}_{1} & =6^{1 / 3} \mathcal{W}_{2}{ }^{2 / 3}
\end{aligned}
$$




$$
\begin{aligned}
& \mathcal{M}_{2}=\mathcal{M}_{3}=\left(\frac{2}{3}\right)^{1 / 3} \mathcal{R}_{2}{ }^{2 / 3} \mathcal{W}_{2}{ }^{2 / 3} \\
& \mathcal{M}_{4}=-6^{-2 / 3} \mathcal{R}_{2} \mathcal{W}_{2}{ }^{2 / 3} \\
& \mathcal{M}_{5}=3^{-2 / 3} \mathcal{R}_{2}{ }^{2 / 3} \mathcal{W}_{2}
\end{aligned}
$$

where

$$
\begin{aligned}
& \mathcal{R}_{2}=3\left(\frac{1}{4} \kappa\left(c^{2} \rho+p\right)\right)^{3} \\
& \mathcal{W}_{2}=-6\left(\frac{c^{2} \kappa}{8 \pi r^{3}}\left(M(r)+M_{g}-\frac{4}{3} \pi r^{3} \rho\right)\right)^{3},
\end{aligned}
$$

we establish that $M_{g}$ is responsible for the conformal curvature singularity ( $M_{g}$ enters $\mathcal{W}_{1}$ and $\mathcal{W}_{2}$ invariants), and does not affect the the regularity of the matter distribution (is absent in $\mathcal{R}_{1}, \mathcal{R}_{2}, \mathcal{R}_{2}$ ). In order to avoid a collapse to Black Hole, the constant $M_{g}$ must be negative. The singularity is naked [8-13] and the gaseous cloud encloses a point-like repulsive gravity source at the center.

\section{The nonrelativistic neutron polytropic approximation}

To build a model, we consider the Fermi gas in nonrelativistic regime with the equation of state approximated by the polytrope [14, 15]

$$
\rho(p)=\frac{1}{c^{2}}\left(\frac{p}{k}\right)^{\frac{1}{\Gamma}}, \quad \Gamma=5 / 3, \quad k=\frac{\hbar^{2}}{15 \pi^{2} m_{N}}\left(\frac{3 \pi^{2}}{c^{2} m_{N}}\right)^{\Gamma} .
$$

We assume that maximal pressure $p_{\max }=p\left(r_{\max }\right)$ does not exceed $10^{34} \mathrm{~Pa}$, as for the regular neutron star. Python language library scipy is used with the vode integrator in order to numerically integrate the system (2)-(3) with the equation of state (12). We adopt the backward differentiation formulas BDF-method (http://www.netlib.org/ode/vode). The central repulsive singularity sweeps the gas out. The region of maximal density forms at some distance $r_{\max }$ from the center. A typical profile is shown in Fig. 1.

The integration was repeated for 59 different values of $r_{\max }$ from the range $r_{\max } \in\left(1 \mathrm{~km}, 10^{2.5} \mathrm{~km}\right)$, and for 10 different $p_{\max } \in\left(5.3 \times 10^{33} \mathrm{~Pa}, 4.6 \times\right.$ $\left.10^{34} \mathrm{~Pa}\right)$ which gives altogether the matrix of 590 profiles. For each profile, the ADM mass, the configuration radius $r_{s}$, the repulsive gravity counterpart $M_{g}$, and the gravitational surface redshift $z$ were found. Details of the integration procedure are explained in [16]. In the set of profiles under investigation, the configuration of the maximal redshift has parameters:

$$
M_{\mathrm{ADM}}=31 M_{\odot}, \quad r_{s}=92 \mathrm{~km}, \quad z=11 .
$$




$$
M_{A D M}=0.98 M_{\odot}, M_{g}=-0.08 M_{\odot}, \quad R=12.54 \mathrm{~km} .
$$

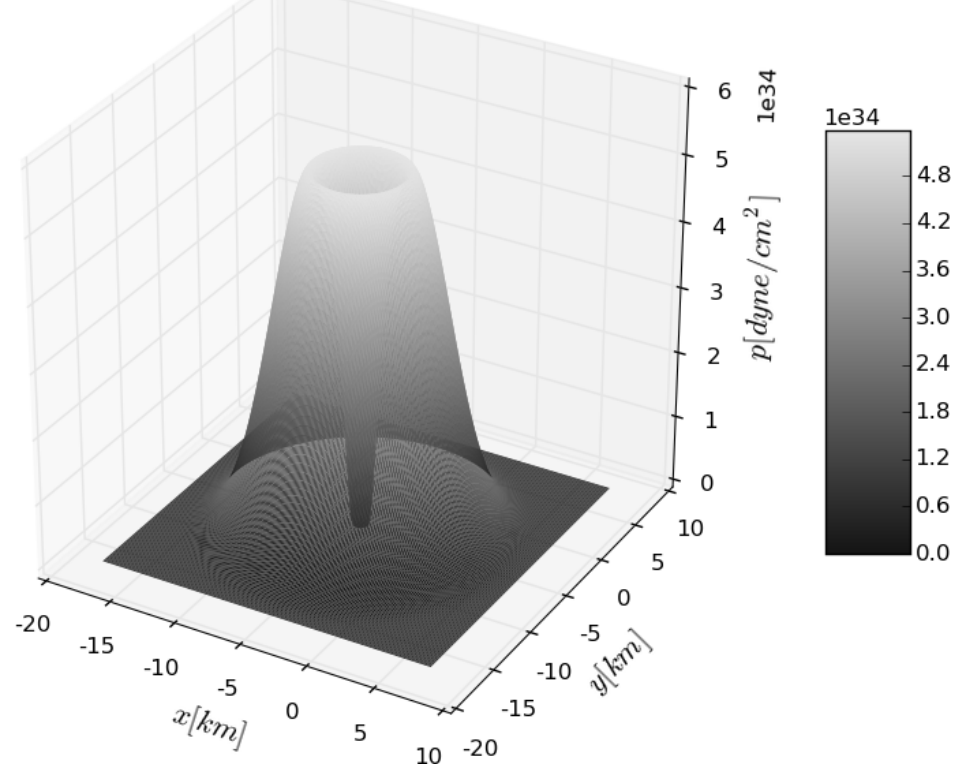

Fig. 1. The pressure profile in $2+1$ dimensional visualization of a typical nakedlysingular, spherical, polytropic cloud.

The maximal value for $z$ (13) comes from the limits for polytrope approximation for the Fermi gas of neutrons and may change with the change of the equation of state. When the regularity conditions for metrics and the cosmic censorship are relaxed, the Oppenheimer limit [4] and the Bondi [17] limit are exceeded. The maximal gravitational redshift we obtained belongs to the range which is presently reserved for cosmology.

\section{REFERENCES}

[1] F.A.E. Pirani, A. Schild, Geometrical and Physical Interpretation of the Weyl Conformal Curvature Tensor, Bull. Acad. Polon. Sci. Sér. Sci. Math. Astronom. Phys. 9, 543 (1961).

[2] R. Penrose, The Road to Reality: A Complete Guide to the Laws of the Universe, Random House, New York 2006.

[3] J. Ponce de Leon, Phys. Rev. 37, 309 (1988).

[4] J.R. Oppenheimer, G.M. Volkoff, Phys. Rev. 55, 374 (1939).

[5] R.M. Wald, General Relativity, University of Chicago Press, Chicago 2010.

[6] J. Carminati, R.G. McLenaghan, J. Math. Phys. 32, 3135 (1991). 
[7] A. Woszczyna et al., http://library.wolfram.com/infocenter/MathSource/8848/

[8] G.W. Gibbons, S.A. Hartnoll, A. Ishibashi, Prog. Theor. Phys. 113, 963 (2005).

[9] A. Krolak, Class. Quantum Grav. 3, 267 (1986).

[10] R. Penrose, J. Asprophys. Astr. 20, 233 (1999).

[11] P.S. Joshi, Gravitational Collapse and Spacetime Singularities, Cambridge Monographs on Mathematical Physics, 2007.

[12] P.S. Joshi, D. Malafarina, R. Narayan, Class. Quantum Grav. 28, 235018 (2011) [arXiv: 1106.5438 [gr-qc]].

[13] P.S. Joshi, D. Malafarina, Int. J. Mod. Phys. D 20, 2641 (2012) [arXiv:1201.3660 [gr-qc]].

[14] R.R. Silbar, S. Reddy, Am. J. Phys. 72, 892 (2004) [Erratum ibid. 73, 286 (2005)] [arXiv:nucl-th/0309041].

[15] J.L. Friedman, J. Astrophys. Astr. 17, 199 (1996).

[16] A. Woszczyna et al., Nakedly Singular Non-vacuum Gravitating Equilibrium States, Gen. Rel. Grav. (accepted).

[17] H. Bondi, Mon. Not. R. Astron. Soc. 302, 337 (1999). 